\title{
Novel Omnidirectional Aerial Manipulator with Elastic Suspension: Dynamic Control and Experimental Performance Assessment
}

\author{
Arda Yiğit, Miguel Arpa Perozo, Loïc Cuvillon, Sylvain Durand and Jacques Gangloff
}

\begin{abstract}
Aerial manipulators may replace industrial manipulators for specific tasks involving large workspaces and high dynamics. However, these robots suffer from a lack of accuracy, autonomy, payload capability and a complex regulatory environment. To overcome some of these limitations, we introduce a novel aerial manipulator suspended by a spring to a robotic carrier. A computed torque controller with exteroceptive feedback is used to control the robot, using singular perturbation theory to prove the stability. We carried out various experiments to assess the workspace, accuracy and dynamics of the manipulator.
\end{abstract}

Index Terms-Aerial Systems: Mechanics and Control, Aerial Systems: Applications, Visual Servoing.

\section{INTRODUCTION}

A ERIAL manipulation consists in using unmanned aerial vehicles (UAVs) to perform robotic manipulation tasks. In that sense, aerial manipulators could be an alternative to industrial manipulators for tasks over a large workspace.

Various configurations are possible in order to deal with the trade-off between energy efficiency and dexterity [1]. Simple tasks such as pick and place can be performed using flying hands, where a gripper is attached to a UAV. For more complex manipulation, the aerial carrier is often equipped with one or more robotic arms.

Aerial robotics, and especially aerial manipulation, is of growing interest in recent years, with major and fast improvements in the area. A major difficulty to overcome is the low energy density of lithium batteries, resulting in low autonomy. Tethered aerial vehicles can be powered externally, and hence have theoretically unlimited flight time [2]. Moreover, maintaining a physical link with the ground saves these robots from legal restrictions, as it is the case in France [3].

Suspending the aerial manipulator is another new concept. In order to optimize its energy consumption and improve its accuracy, SpiderMAV stabilizes itself in a spider-inspired way, launching anchors on nearby ceiling and walls to perch and stabilize the platform [4]. Alternatively, the Suspended Aerial Manipulator (SAM) consists in an omnidirectional UAV, suspended below an aerial carrier by means of cables, and

Manuscript received: October 8, 2020; Accepted December 13, 2020.

This paper was recommended for publication by Editor Pauline Pounds upon evaluation of the Associate Editor and Reviewers' comments. This work was supported by the e-VISER project under grant ANR-17-CE33-0008 by the French National Research Agency (ANR).

The authors are with the ICube Laboratory, University of Strasbourg, INSA Strasbourg, 67000, Strasbourg, France. E-mails: \{arda.yigit, marpaperozo, 1.cuvillon, sdurand, jacques.gangloff \}@unistra.fr

Digital Object Identifier (DOI): see top of this page. carrying a 7-degrees of freedom (DoF) serial manipulator [5]. It is actuated by 8 propellers and 3 winches. The aerial carrier is assumed to be static. The SAM regulates its pose using a cascade control scheme performing proportional-derivative (PD) feedback, with a desired wrench acting on the SAM as control input. A similar system in underwater robotics consists in a hybrid between a cable-driven parallel robot (CDPR) and a thruster-actuated underwater vehicle [6].

Finally, inspired by our previous works [7], [8], where additional embedded thrusters were used to improve the dynamics of planar CDPRs, we developed a novel Aerial Manipulator with Elastic Suspension (AMES) [9]. We proposed an architecture, with only one link, suspending an omnidirectional UAV to a crane-like robotic carrier. A low-stiffness spring, replacing the cable+winch subsystem, provides both mechanical simplicity and reduced energy consumption by compensating for the gravity. The AMES can cover a relatively large workspace (larger than with industrial robots but smaller than with systems using an aerial carrier) with millimetric accuracy and fast dynamics. It could perform various tasks with an adequate carrier: weeding, pesticide delivery or harvesting in association with agricultural robots, pick and place of parcels in logistic hubs with a gantry crane, 3D construction printing in combination with a tower crane.

In this work, we generalize the dynamic model of the AMES to an arbitrary number of propulsion units. A computed torque control law is proposed for the particular case of 6 propulsion units. We use singular perturbation theory to prove the closedloop stability while taking into account the actuator dynamics. Finally, various experiments assess the performances of the AMES. These are our main contributions.

\section{AMES DESCRIPTION}

We designed the AMES as shown in Fig. 1, where an aerial manipulator is suspended at the tip of a crane-like robotic carrier by a spring. The aerial manipulator, which is called here an Aerial Wrench Generator (AWG), is holding a gripper. The purpose of the AWG is to generate a 6-DoF wrench at the end effector of the AMES thanks to propulsion units. So the AMES with its robotic carrier, spring and AWG may be considered as a new type of robot: a hybrid between a serial manipulator and an aerial manipulator with a spring acting as a flexible linkage.

The spring compensates for the gravity, so the AWG is almost free-floating in the air around its equilibrium position, 
and therefore, unlike a UAV, requires very little energy. Note that it may be possible to suspend and power the robot with a coil cord. The spring stiffness has to be as low as possible to maximize the workspace of the AWG. Moreover, there is no more need to use an actuated winch to control the altitude.

The robotic carrier is optional. It aims to move the equilibrium position of the AWG slowly to the average position of the current task, reducing even more the energy consumption, and increasing the workspace.

Since the gripper does not provide any mobility other than the gripping, the AWG must be able to generate a 6-DoF wrench to control the pose of the end effector. Such an AWG can be designed using either unidirectional or bidirectional propulsion units [10]. For the bidirectional case, it is possible to have only 6 propulsion units, whereas for the unidirectional case, at least 7 are needed [11].

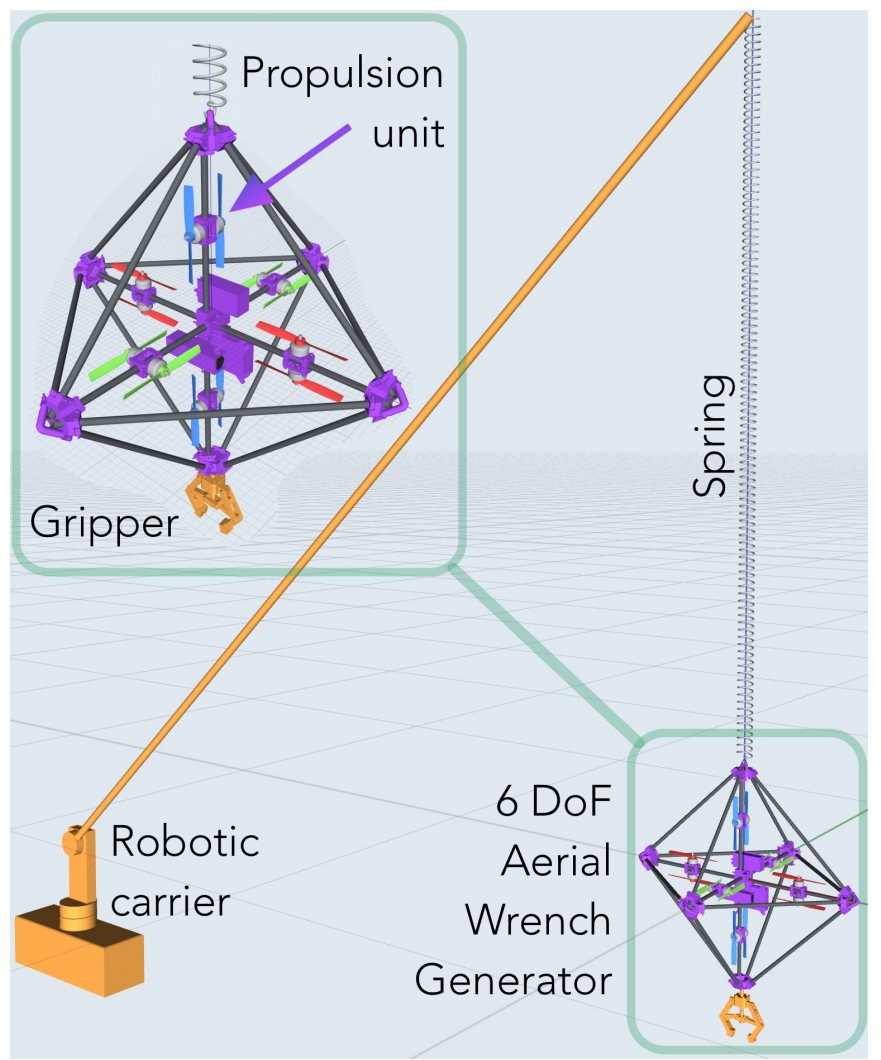

Fig. 1. AMES concept.

\section{MODELING}

In this section, the dynamic model of the AMES is derived. The AWG is suspended by a massless elastic link. The robotic carrier is supposed to be much slower than the AWG, and hence, in this study, the spring anchoring point is considered static. The mass of the load is assumed to be negligible.

\section{A. System parametrization and notations}

The geometric parameters of the system are shown in Fig. 2 In order to be as generic as possible, the following model makes no assumptions on the number of propulsion units

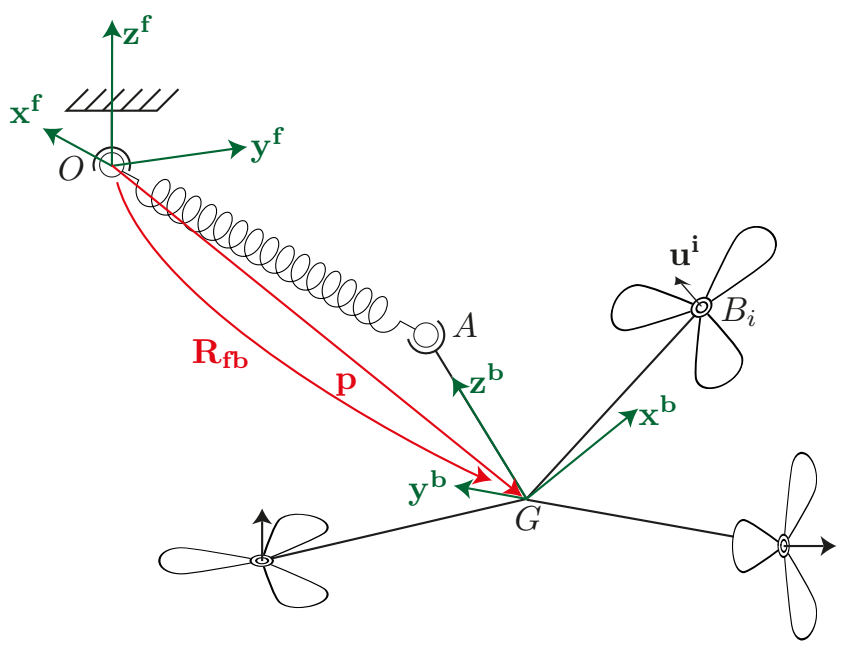

Fig. 2. Geometric parametrization of the i-th propulsion unit.

(can be under- or over-actuated), nor on their directionality (unidirectional or bidirectional).

The origin $O$ of the inertial frame $\mathcal{R}_{f}=\left(O, \mathbf{x}^{\mathbf{f}}, \mathbf{y}^{\mathbf{f}}, \mathbf{z}^{\mathbf{f}}\right)$ is the static anchoring point of the spring, while $A$ is its mobile end. The body frame $\mathcal{R}_{b}=\left(G, \mathbf{x}^{\mathbf{b}}, \mathbf{y}^{\mathbf{b}}, \mathbf{z}^{\mathbf{b}}\right)$ is positioned at the center of mass (CoM) $G$ of the AWG, its $\mathbf{z}^{\mathbf{b}}$ axis points toward $A$. The rotation matrix $\mathbf{R}_{\mathrm{fb}} \in S O(3)$ describes the orientation of $\mathcal{R}_{b}$ with respect to $\mathcal{R}_{f}$. The AWG has $n$ propulsion units, positioned in an arbitrary way. The position of the center of the $i$-th propulsion unit is $B_{i}$, and $\mathbf{u}^{\mathbf{i}}$ is the thrust direction. The position vector of the $\operatorname{CoM} G$ is $\mathbf{p}$.

Let $\mathbf{u}$ and $\mathbf{v}$ be vectors and $\mathcal{R}_{q}$ a reference frame. The projection of $\mathbf{v}$ in $\mathcal{R}_{q}$ is written $\mathbf{v}_{q}$. The cross product of $\mathbf{u}_{q}$ and $\mathbf{v}_{q}$ is denoted $\mathbf{u}_{q} \times \mathbf{v}_{q}$ and $[\cdot]_{\times}$is the cross product matrix such that $\mathbf{u}_{q} \times \mathbf{v}_{q}=\left[\mathbf{u}_{q}\right]_{\times} \mathbf{v}_{q}$.

\section{B. AWG dynamics}

We assume the AWG dynamics can be approximated by a single-body dynamic model, which means the inertiae of the rotating parts (rotor and propeller) of a propulsion unit have a negligible influence on the global dynamics. Thereby, the AWG dynamics can be obtained using Newton-Euler's equations:

$$
\begin{array}{r}
\left(\begin{array}{cc}
m \mathbf{I}_{3} & \mathbf{0}_{3} \\
\mathbf{0}_{3} & \mathbf{J}_{b}
\end{array}\right)\left(\begin{array}{c}
\ddot{\mathbf{p}}_{f} \\
\dot{\boldsymbol{\omega}}_{b}
\end{array}\right)+\left(\begin{array}{c}
\mathbf{0}_{3 \times 1} \\
\boldsymbol{\omega}_{b} \times \mathbf{J}_{b} \boldsymbol{\omega}_{b}
\end{array}\right)+\left(\begin{array}{c}
-m \mathbf{g}_{f} \\
\mathbf{0}_{3 \times 1}
\end{array}\right) \\
-\left(\begin{array}{c}
\mathbf{F}_{f}^{\mathbf{s}} \\
\mathbf{N}_{b}^{\mathbf{s}}
\end{array}\right)=\left(\begin{array}{c}
\mathbf{F}_{f} \\
\mathbf{N}_{b}
\end{array}\right)
\end{array}
$$

with $m>0$ the total mass of the platform, $\mathbf{J}_{b} \in \mathbb{R}^{3 \times 3}$ its inertia tensor at the CoM expressed in $\mathcal{R}_{b}, \boldsymbol{\omega}_{b} \in \mathbb{R}^{3}$ the angular velocity with respect to $\mathcal{R}_{f}, \mathrm{~g}_{f} \in \mathbb{R}^{3}$ the gravity acceleration, $\mathbf{F}_{f}^{\mathbf{s}} \in \mathbb{R}^{3}$ the force of the elastic link on the AWG and $\mathbf{N}_{b}^{\mathbf{s}} \in$ $\mathbb{R}^{3}$ the associated torque at the CoM, $\mathbf{F}_{f} \in \mathbb{R}^{3}$ the resulting force generated by the propellers and $\mathbf{N}_{b} \in \mathbb{R}^{3}$ the associated torque at the CoM.

The behavior of the elastic link is modeled as a simple linear spring with stiffness $k$ and free length $l_{0}$ :

$$
\mathbf{F}_{f}^{\mathbf{s}}=-k\left(\|\mathbf{O A}\|-l_{0}\right) \frac{\mathbf{O} \mathbf{A}_{f}}{\|\mathbf{O A}\|}
$$


where

$$
\mathbf{O A}_{f}=\mathbf{p}_{f}+\|\mathbf{G A}\| \mathbf{R}_{\mathbf{f b}} \mathbf{z}_{b}^{\mathbf{b}}
$$

Therefore, the associated torque is

$$
\mathbf{N}_{b}^{\mathbf{s}}=\|\mathbf{G A}\| \mathbf{z}_{b}^{\mathbf{b}} \times \mathbf{R}_{\mathbf{f b}}^{T} \mathbf{F}_{f}^{\mathbf{s}}
$$

The thrust and the drag generated by a propeller are proportional to the squared rotational speed of the propeller and oriented along the propeller axis [12]. In the following, $a$ (in $\left[\mathrm{N} \mathrm{rad}^{-2} \mathrm{~s}^{2}\right]$ ) denotes the thrust coefficient and $b$ (in $\left.\left[\mathrm{N} \mathrm{m} \mathrm{rad}{ }^{-2} \mathrm{~s}^{2}\right]\right)$ the drag coefficient.

Let $\mathbf{W}_{b} \in \mathbb{R}^{6 \times n}$ be the matrix that maps the column matrix $\mathbf{w}_{2}=\left(\cdots w_{i}\left|w_{i}\right| \cdots\right)^{T}$ of signed squared propeller rotational velocities $w_{i}$ to the wrench they apply on the platform expressed in the body frame $\mathcal{R}_{b}$ :

$$
\left(\begin{array}{l}
\mathbf{F}_{b} \\
\mathbf{N}_{b}
\end{array}\right)=\mathbf{W}_{b} \mathbf{w}_{2}
$$

$$
\mathbf{W}_{b}=a\left(\begin{array}{ccc}
\cdots & \mathbf{u}_{b}^{\mathbf{i}} & \cdots \\
\cdots & \mathbf{G B}_{b}^{\mathbf{i}} \times \mathbf{u}_{b}^{\mathbf{i}} & \cdots
\end{array}\right)+b\left(\begin{array}{ccc}
\cdots & \mathbf{0}_{3 \times 1} & \cdots \\
\cdots & \mathbf{u}_{b}^{\mathbf{i}} & \cdots
\end{array}\right)
$$

Let $\mathbf{X}=\left(\mathbf{p}^{T} \boldsymbol{\eta}^{T}\right)^{T}$ be the pose coordinates of the AWG, with $\mathbf{p}=\mathbf{p}_{f}$ the position of the CoM in the fixed frame and $\boldsymbol{\eta}=(\psi \theta \phi)^{T}$ a set of Euler angles. The so-called analytical Jacobian matrix $\mathbf{S}(\boldsymbol{\eta}) \in \mathbb{R}^{3 \times 3}$ maps the time derivative of $\boldsymbol{\eta}$ to the angular velocity $\boldsymbol{\omega}_{b}$ :

$$
\boldsymbol{\omega}_{b}=\mathbf{S}(\boldsymbol{\eta}) \dot{\boldsymbol{\eta}}
$$

Combining (1), (2) and (3), the equations of motion of the robot can be written in the operational-space canonical formulation:

$$
\begin{aligned}
& \underbrace{\left(\begin{array}{cc}
m \mathbf{I}_{3} & \mathbf{0}_{3} \\
\mathbf{0}_{3} & \mathbf{S}^{T} \mathbf{J}_{b} \mathbf{S}
\end{array}\right)}_{\mathbf{M}(\mathbf{X})}\left(\begin{array}{l}
\ddot{\mathbf{p}} \\
\ddot{\boldsymbol{\eta}}
\end{array}\right)+\underbrace{\left(\begin{array}{cc}
\mathbf{0}_{3} & \mathbf{0}_{3} \\
\mathbf{0}_{3} & \mathbf{S}^{T}\left(\mathbf{J}_{b} \dot{\mathbf{S}}+[\mathbf{S} \dot{\boldsymbol{\eta}}]_{\times} \mathbf{J}_{b} \mathbf{S}\right)
\end{array}\right)}_{\mathbf{C}(\mathbf{X} \dot{\mathbf{X}})}\left(\begin{array}{l}
\dot{\mathbf{p}} \\
\dot{\boldsymbol{\eta}}
\end{array}\right) \\
& +\underbrace{\left(\begin{array}{c}
-m \mathbf{g}_{f}-\mathbf{F}_{f}^{\mathbf{s}} \\
-\mathbf{S}^{T} \mathbf{N}_{b}^{\mathbf{s}}
\end{array}\right)}_{\mathbf{G}(\mathbf{X})}=\underbrace{\left(\begin{array}{cc}
\mathbf{R}_{\mathbf{f b}} & \mathbf{0}_{3} \\
\mathbf{0}_{3} & \mathbf{S}^{T}
\end{array}\right) \mathbf{W}_{b}}_{\tilde{\mathbf{W}}(\mathbf{X})} \mathbf{w}_{\mathbf{2}}
\end{aligned}
$$

\section{COMPUTED TORQUE CONTROLLER}

To track a desired trajectory with the AWG, a computed torque control law [13] is used. An inverse model of the AWG dynamics provides a linearized model of the AWG suitable for a simple PD regulation.

\section{A. Control law}

Let us recall the dynamic model (4) in its canonical form:

$$
\mathbf{M}(\mathbf{X}) \ddot{\mathbf{X}}+\mathbf{C}(\mathbf{X}, \dot{\mathbf{X}}) \dot{\mathbf{X}}+\mathbf{G}(\mathbf{X})=\tilde{\mathbf{W}}(\mathbf{X}) \mathbf{w}_{2}
$$

where $\tilde{\mathbf{W}}$ is always invertible since the AWG's actuators are not redundant and there is no singular configuration.

To follow a desired trajectory $\mathbf{X}^{*}$, the following computed torque control law is applied:

$$
\hat{\mathbf{W}} \mathbf{w}_{2}=\hat{\mathbf{M}}\left(\ddot{\mathbf{X}}^{*}+\mathbf{u}\right)+\hat{\mathbf{C}} \dot{\mathbf{X}}+\hat{\mathbf{G}}
$$

with $\mathbf{u}$ a feedback control input, $\hat{\mathbf{W}}, \hat{\mathbf{M}}, \hat{\mathbf{C}}$ and $\hat{\mathbf{G}}$ are the estimates of $\tilde{\mathbf{W}}, \mathbf{M}, \mathbf{C}$ and $\mathbf{G}$ in (5).

The signal $\mathbf{u}$ corresponds here to a Proportional-Derivative (PD) controller: $\mathbf{u}=\mathbf{k}_{\mathbf{d}} \dot{\mathbf{e}}+\mathbf{k}_{\mathbf{p}} \mathbf{e}$, with $\mathbf{e}=\mathbf{X}^{*}-\mathbf{X}$ the pose error signal, $\mathbf{k}_{\mathbf{p}}$ and $\mathbf{k}_{\mathbf{d}}$ proportional and derivative definite positive gain matrices tuned for disturbance rejection and desired closed-loop dynamic performances. Thus, the controller output is:

$$
\mathbf{w}_{2}=\hat{\mathbf{W}}^{-1}\left(\hat{\mathbf{M}}\left(\ddot{\mathbf{X}}^{*}+\mathbf{k}_{\mathbf{d}} \dot{\mathbf{e}}+\mathbf{k}_{\mathbf{p}} \mathbf{e}\right)+\hat{\mathbf{C}} \dot{\mathbf{X}}+\hat{\mathbf{G}}\right)
$$

\section{B. Stability analysis}

In a classical computed torque, as its name indicates, the output of the controller is directly the torque or force input reference of the actuators. When dealing with standard UAV electronic speed controllers (ESCs), this is not possible. Instead, the input signal of the ESCs is proportional to the armature voltage of an equivalent DC motor of the propeller actuator. Since propeller thrust is related to its squared rotational velocity, implementing a low-level velocity loop at the actuator level allows for a force control of the robot. However, taking into account the actuator dynamics, stability of the computed torque control law is no more guaranteed. A similar problem is the control of a CDPR via feedback linearization [14], when a position loop is implemented at the winch-actuator level. The stability was proven by means of singular perturbation theory.

We propose a similar approach to prove the stability of the closed-loop system. We assume that the propeller rotational velocity control loop is significantly faster than the AWG dynamics and a first-order system with time constant $t_{m}$ models the evolution of the actual velocity $\mathbf{w}$ according to the desired velocity $\mathbf{w}^{*}$.

Let us introduce the fast variable $\mathbf{z}=\mathbf{w}, \varepsilon=t_{m}$ a small quantity and

$$
\mathrm{x}=\left(\begin{array}{c}
\mathrm{x}_{1} \\
\mathrm{x}_{2}
\end{array}\right)=\left(\begin{array}{l}
\mathrm{X} \\
\dot{\mathrm{X}}
\end{array}\right)
$$

The standard singular perturbation model [15] of the AWG and its actuator dynamics is written using these new variables:

$$
\begin{aligned}
& \dot{\mathbf{x}}=\left(\begin{array}{c}
\mathbf{x}_{\mathbf{2}} \\
\mathbf{M}\left(\mathbf{x}_{1}\right)^{-1}\left(\tilde{\mathbf{W}}\left(\mathbf{x}_{1}\right) \mathbf{z}_{2}-\mathbf{C}\left(\mathbf{x}_{1}, \mathbf{x}_{2}\right) \mathbf{x}_{2}-\mathbf{G}\left(\mathbf{x}_{1}\right)\right)
\end{array}\right) \\
& \varepsilon \dot{\mathbf{z}}=\mathrm{w}^{*}-\mathrm{z}
\end{aligned}
$$

with $\mathbf{z}_{2}=\left(\cdots z_{i}\left|z_{i}\right| \cdots\right)^{T}$.

Setting $\varepsilon=0$ in (9) gives the unique quasi-steady state of the fast dynamics:

$$
\mathbf{h}(t, \mathbf{x})=\mathbf{w}^{*}
$$

If the modeling errors are negligible, combining (7), (8) and (10) for $\varepsilon=t_{m}=0$ gives the reduced model:

$$
\dot{\mathbf{x}}=\left(\begin{array}{c}
\mathbf{x}_{\mathbf{2}} \\
\ddot{\mathbf{X}}^{*}+\mathbf{k}_{\mathbf{d}}\left(\dot{\mathbf{X}}^{*}-\mathbf{x}_{\mathbf{2}}\right)+\mathbf{k}_{\mathbf{p}}\left(\mathbf{X}^{*}-\mathbf{x}_{\mathbf{1}}\right)
\end{array}\right)
$$

Equivalently, the reduced model can be written using the pose error $\mathbf{e}=\mathbf{X}^{*}-\mathbf{x}_{\mathbf{1}}$ :

$$
\left(\begin{array}{c}
\dot{\mathbf{e}} \\
\ddot{\mathbf{e}}
\end{array}\right)=\left(\begin{array}{cc}
0 & \mathbf{I}_{3 \times 3} \\
-\mathbf{k}_{\mathbf{p}} & -\mathbf{k}_{\mathbf{d}}
\end{array}\right)\left(\begin{array}{l}
\mathbf{e} \\
\dot{\mathbf{e}}
\end{array}\right)
$$


To prove the stability of the reduced model, let us define the following Lyapunov candidate function: $V=\frac{1}{2} \dot{\mathbf{e}}^{T} \dot{\mathbf{e}}+\frac{1}{2} \mathbf{e}^{T} \mathbf{k}_{\mathbf{p}} \mathbf{e}$. Its time derivative is $\dot{V}=-\dot{\mathbf{e}}^{T} \mathbf{k}_{\mathbf{d}} \dot{\mathbf{e}}$. As $\mathbf{k}_{\mathbf{d}}$ and $\mathbf{k}_{\mathbf{p}}$ are both positive definite matrices, $V>0$ and $\dot{V} \leq 0$. Invoking LaSalle's theorem [15], the error tends towards zero: the reduced model is asymptotically stable at the origin, and since it is linear, it is also exponentially stable.

Let us introduce the "stretched" time variable $t_{\varepsilon}=t / \varepsilon$. Then, the boundary-layer model is:

$$
\frac{d \mathbf{y}}{d t_{\varepsilon}}=-\mathbf{y}
$$

with $\mathbf{y}=\mathbf{z}-\mathbf{h}(t, \mathbf{x})=\mathbf{w}-\mathbf{w}^{*}$. The boundary-layer model (13) is an exponentially stable first-order system.

Since the reduced model (12) and the boundary-layer model (13) are both exponentially stable at the origin and if $\mathbf{X}^{*}$ is a feasible trajectory (continuous and has bounded derivative), Tikhonov's theorem (theorem 11.2 from [15]) states that the solution of the standard singular perturbation model $\mathbf{x}$ approaches the solution of the reduced model $\overline{\mathbf{x}}$ : for $\varepsilon=t_{m}$ small enough, the singular perturbation problem has a unique solution that verifies:

$$
\mathbf{x}(t, \varepsilon)-\overline{\mathbf{x}}(t)=O(\varepsilon)
$$

Therefore, the closed-loop system is stable if the time constant $t_{m}$ of the propeller dynamics is small enough.

\section{EXPERIMENTAL SETUP}

This section presents the experimental setup, with the main focus on the design of a prototype named "dextAIR".

\section{A. Mechanical design}

The AWG has the same structure as an omnidirectional multirotor vehicle with bidirectional thrusters described in [16]. The relative placement of propulsion units is optimized to maximize the agility, i.e. the highest thrust and torque that can be generated in all directions. We selected the optimal configuration for $n=6$ propulsion units (Fig. 3a in [16]). This configuration can generate a 6-DoF wrench without overactuation, simplifying the thrust allocation problem. With this configuration, the propulsion units are attached to the vertices of a regular octahedron. As a consequence, dextAIR is as rotationally invariant as possible and its inertia matrix is close to the identity matrix.

Depending on its mass, the transverse vibrations of the spring can significantly disturb the AWG. That can be avoided by mounting the spring horizontally and suspending the AWG by a cable through a pulley (see Fig. 3.

Some ESCs can drive motors in both directions. Combined with symmetrical propellers (also called 3D propellers), one can use them to generate force in both directions. However, as shown in [17], if a change of rotation direction occurs, the settling time to reach the new propeller velocity is longer than the settling time of a velocity reference without direction change. This propeller dynamics degradation and nonlinearity results from the crossing of the zero velocity of the propeller. Indeed, ESCs use the back EMF (electromotive force) to measure the speed of the rotor, which is not possible at low speeds. For a standard multirotor drone, this may not be an important issue since there is no equilibrium point and propellers need to generate a force constantly in order to hover, but it is a limiting factor for the dextAIR. Indeed, propellers may need to change the rotation direction many times per second (for example, the natural frequency for roll/pitch angles of the robot is higher than $3 \mathrm{~Hz}$ ).

Each propulsion unit (Fig. 3) is made of a pair of propellers (DALPROP 5045), mounted on two coaxial brushless DC motors (T-Motor F-40 Pro III Kv2400), in order to be able to generate a force in both directions without changing direction of rotation, avoiding the "zero-crossing" problem. According to the sign of the force to be generated, the corresponding propeller rotates at the desired speed while the second one is idling, i.e. rotating at its lowest speed $(1500 \mathrm{rpm}$, equivalent to $0.03 \mathrm{~N}$ ).

\section{B. Electronics}

Each propeller is driven by a brushless DC motor. The computed torque controller computes the desired speed of the propellers. However, there are only a limited number of commercial ESCs providing speed regulation and most of them are designed for helicopters and do not have fast dynamics. The ESC firmware can be modified to add speed regulation using an external tachometer [16]. A sensorless solution also exists for BL-Ctrl boards [18]. We developed the open-source Teensyshot firmware (https://github.com/jacqu/teensyshot) for the speed control of brushless DC motors without additional sensors. Teensyshot can be used with up to 6 ESCs compatible with the DSHOT600 protocol. A fast PID (proportionalintegral-derivative) speed regulation loop, using real-time ESC telemetry data acquired through 115200 bps serial link, runs at $500 \mathrm{~Hz}$ on a Teensy 4.0 micro-controller board. KISS 32A ESCs are used for the experiments.

The AWG is autonomous: it carries its own energy source, a $2300 \mathrm{~mA} \mathrm{~h}, 11.1 \mathrm{~V} / 3 \mathrm{~S}$ lithium polymer battery pack (TATTU 3S1P). It also has an on-board CPU (Raspberry Pi 4B) running high-level control algorithms and communicating with a ground station through Wi-Fi TCP/IP sockets thanks to the open-source Simulink toolbox RPIt developed in our lab [19]. The Raspberry Pi is connected by USB to 2 Teensy boards regulating the velocity of a total of 12 propellers.

The pose and the twist of the AWG are acquired by a Vicon Bonita motion-capture system (see Fig. 3) measuring the 6DoF pose vector of the AWG with a refresh rate of $240 \mathrm{~Hz}$ and a gyroscopic sensor (MPU-9150).

\section{Controller tuning}

In addition to the gyroscopic effect, the drag is also neglected for the experiments. Indeed, the maximum drag torque that can be generated with a single propulsion unit is approximately $0.1 \mathrm{Nm}$ and the maximum gyroscopic torque when the AWG rotates at $2 \pi \mathrm{rad} \mathrm{s}^{-1}$ is approximately $0.4 \mathrm{~N} \mathrm{~m}$, both significantly smaller than $2.4 \mathrm{Nm}$, the maximum torque that the AWG can attain. Thereby, propulsion units are considered as pure force generators. 


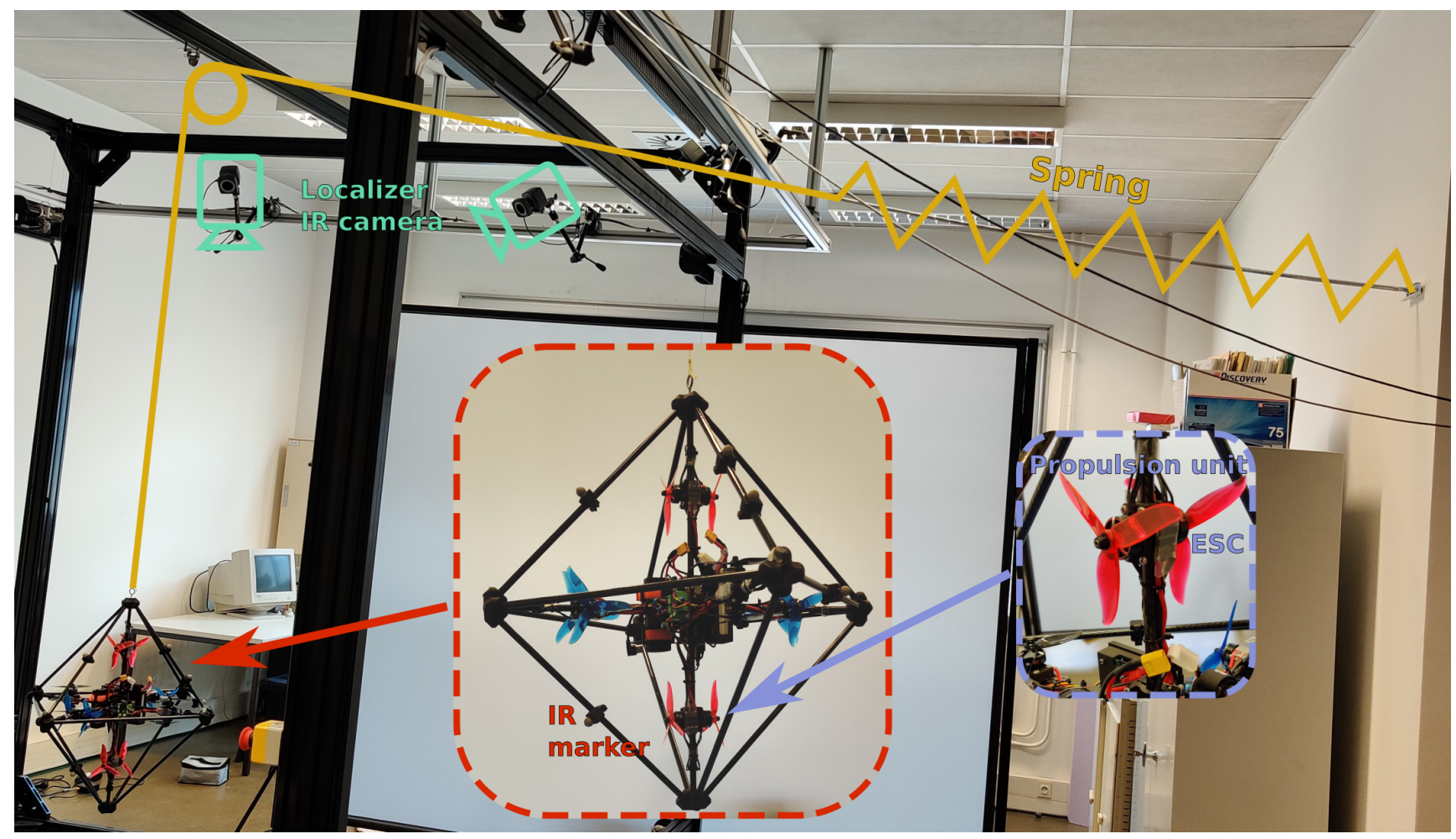

Fig. 3. dextAIR prototype.

The propeller speed control loop is tuned such that, in closed loop, it behaves like a first-order system with $25 \mathrm{~ms}$ time constant.

The computed torque controller of the AMES runs at $200 \mathrm{~Hz}$ frequency. An integral term is added to eliminate the steadystate error, compensating for small unmodeled phenomena:

$$
\tau=\hat{\mathbf{M}}\left(\ddot{\mathbf{x}}_{\mathbf{d}}+\mathbf{k}_{\mathbf{d}} \dot{\mathbf{e}}+\mathbf{k}_{\mathbf{p}} \mathbf{e}+\mathbf{k}_{\mathbf{i}} \int \mathbf{e}\right)+\hat{\mathbf{C}} \dot{\mathbf{x}}+\hat{\mathbf{G}}
$$

For the experiments, roll-pitch-yaw angles describe the orientation of the AWG. The PID gain matrices are diagonal, therefore the control of all DoFs is decoupled:

$$
\begin{aligned}
\mathbf{k}_{\mathbf{p}} & =\operatorname{diag}(150,150,150,150,150,120) \\
\mathbf{k}_{\mathbf{d}} & =\operatorname{diag}(20,20,20,20,20,20) \\
\mathbf{k}_{\mathbf{i}} & =\operatorname{diag}(500,500,500,500,500,500)
\end{aligned}
$$

The model parameters are presented in Table I

TABLE I

MODEL PARAMETERS

\begin{tabular}{llr}
\hline Parameter & Description & Value \\
\hline$m$ & Total mass & $1.95 \mathrm{~kg}$ \\
$J_{x}$ & Moments of inertia about $\mathbf{x}^{\mathbf{b}}$ & $3.53 \times 10^{-3} \mathrm{~kg} \mathrm{~m}^{2}$ \\
$J_{y}$ & Moments of inertia about $\mathbf{y}^{\mathbf{b}}$ & $3.44 \times 10^{-3} \mathrm{~kg} \mathrm{~m}^{2}$ \\
$J_{z}$ & Moments of inertia about $\mathbf{z}^{\mathbf{b}}$ & $3.40 \times 10^{-3} \mathrm{~kg} \mathrm{~m}^{2}$ \\
$k$ & Spring stiffness & $22 \mathrm{~N} \mathrm{~m}^{-1}$ \\
$l_{0}$ & Spring free length & $0.78 \mathrm{~m}$ \\
$L$ & Propeller axis to CoM distance & $0.17 \mathrm{~m}$ \\
$\|$ GA $\|$ & Spring to CoM distance & $0.345 \mathrm{~m}$ \\
$a$ & Thrust coefficient & $1.36 \mu \mathrm{Nad}^{-2} \mathrm{~s}^{2}$ \\
$F_{\text {max }}$ & Maximum thrust & $7.2 \mathrm{~N}$
\end{tabular}

\section{PERFORMANCE CHARACTERISTICS}

Numerous tests are carried out to assess the performance of the robot. This section presents static, dynamic and energy performances of the AMES. A trajectory following experiment is available in the video associated to the paper (https://youtu.be/Ey88RkaMrNc).

\section{A. Static performance}

1) Workspace: The workspace of the AMES depends mainly on the robotic carrier. However the motion of the carrier is supposed to be slow, so its fast dynamic workspace is restricted around the equilibrium point.

The Total Orientation Workspace (TOW) is the set of reachable positions for any orientation in a given set [20]. Since there is no kinematic constraints on the robot, this workspace is only limited by the spring and motor saturations, and can be disclosed by discretizing the 3D space.

The TOW for a $5^{\circ}$ tilt is illustrated in Fig. 4, in green the theoretical one obtained in simulations by discretizing the 3D space, and in red a cuboid whose vertices are tested experimentally. For the sake of simplicity, the experimental validation is done by testing if the robot can reach a $5^{\circ}$ roll and pitch independently. Because of the geometry of the robot, the TOW has two planes of symmetry $(O x z)$ and $(O y z)$. The theoretical workspace volume is equivalent to the volume of a cube with a $0.65 \mathrm{~m}$ edge while the practical one is equivalent to a cube with only a $0.50 \mathrm{~m}$ edge. A more accurate model of the spring and parameter estimation procedure would be required to reduce the discrepancy between these two workspaces. 
To extend the TOW, it is possible to optimize further the design of the AWG. If the on-board anchoring point is at the CoM, that is $\|\mathbf{G A}\|=0$, there is no elastic restoring torque to compensate for. Another possibility consists in increasing the distance between the propeller axis and the CoM, $L$, to be able to generate a higher torque, but this will deteriorate angular dynamics by increasing the inertia. With both strategies, the theoretical workspace could have a volume equivalent to a cube with a $1.86 \mathrm{~m}$ edge, with the current spring stiffness $k$.

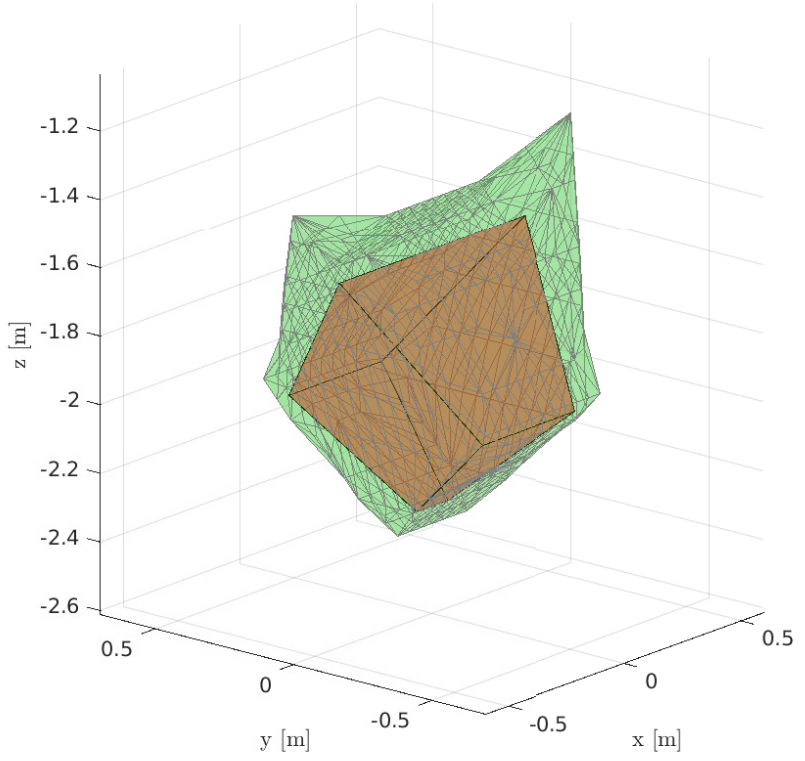

Fig. 4. Total Orientation Workspace: theoretical (green) and tested (red).

2) Pose characteristics: Performance characteristics and test methods for industrial robots are defined by the ISO 9283 standard [21].

For each operational coordinate $q \in \mathbf{x}$, the pose accuracy $A P_{q}$ measures the gap between the desired pose $q_{c}$ and the mean of the attained poses $\bar{q}$ (after repeating the same pose $N$ times):

$$
A P_{q}=\left(\bar{q}-q_{c}\right)
$$

The positioning accuracy $A P_{P}$ is the distance between the desired position and the barycenter of the attained positions: $A P_{P}=\sqrt{A P_{x}^{2}+A P_{y}^{2}+A P_{z}^{2}}$.

The pose repeatability $R P$ measures the disparity between attained poses. The positioning repeatability $R P_{l}$ is the sum of the average position errors $\bar{l} \geq 0$ and 3 times their standard deviation $S_{l}$ :

$$
R P_{l}=\bar{l}+3 S_{l}
$$

For each orientation coordinate $\alpha \in \boldsymbol{\eta}$, the orientation repeatability $R P_{\alpha}$ is 3 times the standard deviation of the errors on $\alpha$ :

$$
R P_{\alpha}=3 S_{\alpha}
$$

The tests are done in a subset of the workspace: a cube centered at the equilibrium point of the robot, with edges parallel to $\mathcal{R}_{f}$ axes and of side length $S=0.4 \mathrm{~m}$ (see Fig. 5). The positions $P_{1}$ to $P_{5}$ are selected according to the ISO 9283 standard. The robot performed $N=10$ cycles from $P_{5}$ to $P_{1}$, with a maximum speed of $V_{M}=0.63 \mathrm{~m} \mathrm{~s}^{-1}$ and with a constant null orientation.

The results for the accuracy and repeatability tests are summarized in Table [II. For the positioning, the robot has a submillimetric accuracy and repeatability lower than $2 \mathrm{~mm}$. For the orientation, both the accuracy and the repeatability are lower than $1^{\circ}$. The yaw repeatability, however, is more sensitive than for roll and pitch.

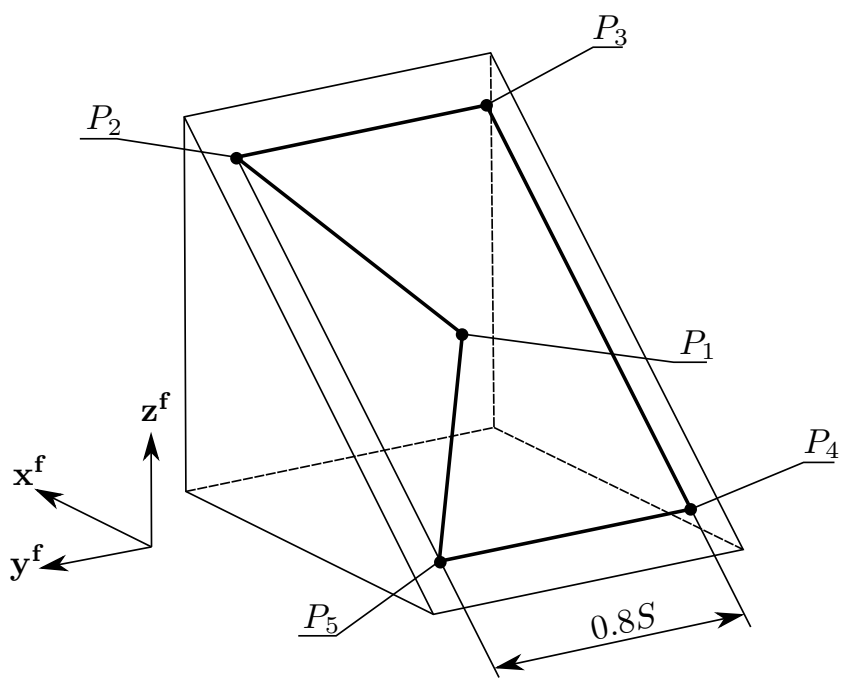

Fig. 5. Path $\left(P_{5}\right.$ to $\left.P_{1}\right)$.

TABLE II

ACCURACY AND REPEATABILITY

\begin{tabular}{lrrrrr}
\hline Positions & $P_{1}$ & $P_{2}$ & $P_{3}$ & $P_{4}$ & $P_{5}$ \\
\hline$A P_{P}[\mathrm{~mm}]$ & 0.14 & 0.08 & 0.11 & 0.10 & 0.19 \\
$R P_{l}[\mathrm{~mm}]$ & 0.48 & 1.49 & 0.57 & 1.05 & 1.73 \\
$A P_{\psi}\left[^{\circ}\right]$ & 0.00 & -0.04 & -0.01 & -0.02 & 0.01 \\
$R P_{\psi}\left[^{\circ}\right]$ & 0.16 & 0.61 & 0.30 & 0.42 & 0.39 \\
$A P_{\theta}\left[^{\circ}\right]$ & -0.00 & -0.01 & 0.01 & -0.01 & 0.03 \\
$R P_{\theta}\left[^{\circ}\right]$ & 0.13 & 0.43 & 0.16 & 0.29 & 0.42 \\
$A P_{\phi}\left[^{\circ}\right]$ & 0.00 & -0.02 & -0.02 & -0.01 & -0.01 \\
$R P_{\phi}\left[^{\circ}\right]$ & 0.27 & 0.56 & 0.30 & 0.31 & 0.57
\end{tabular}

\section{B. Dynamic performance}

1) Step response: Table III summarizes the step responses of the system for each independent DoF. These step responses are shown on Fig. 6 and 7 , where $\delta z_{\text {ref }}$ and $\delta z$ denote respectively the desired and actual positions along the vertical axis relatively to the equilibrium position.

The step responses illustrate the high dynamics of the AWG. The rise time from $10 \%$ to $90 \%$ is less than $100 \mathrm{~ms}$ for a position step and less than $200 \mathrm{~ms}$ for an orientation step. The overshoot can be up to $70 \%$, but is acceptable since practical trajectories are smoother and therefore cause less overshoot. The steady-state error is eliminated, but small orientation oscillations persist due to measurement noise. A slight coupling between DoFs during transients persists due to the neglected reaction torque of the propulsion units.

2) Maximum acceleration: To measure the maximum acceleration of the robot, a step reference that saturates the actuators is used. Since the second derivative of the position 

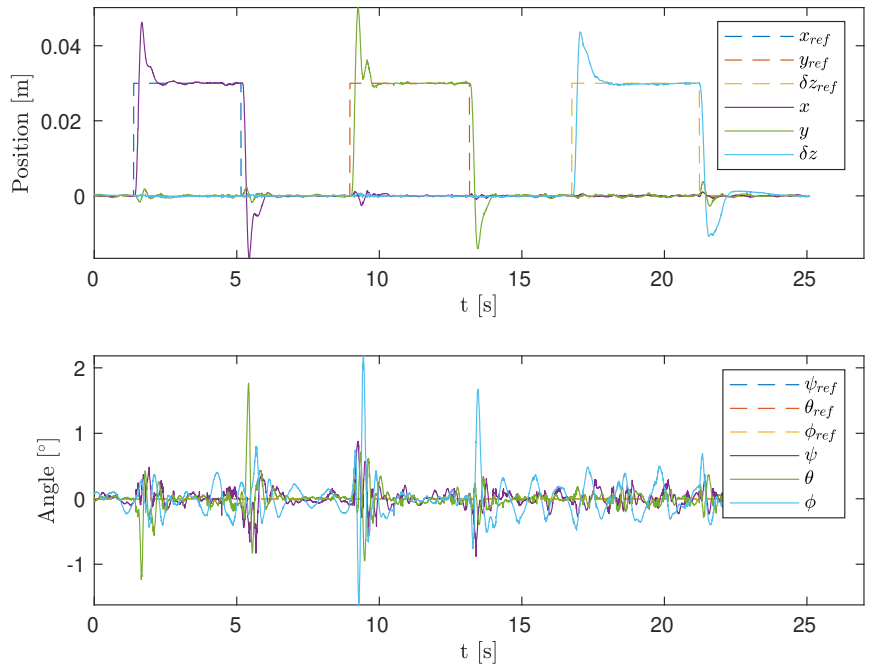

Fig. 6. Response to position steps.
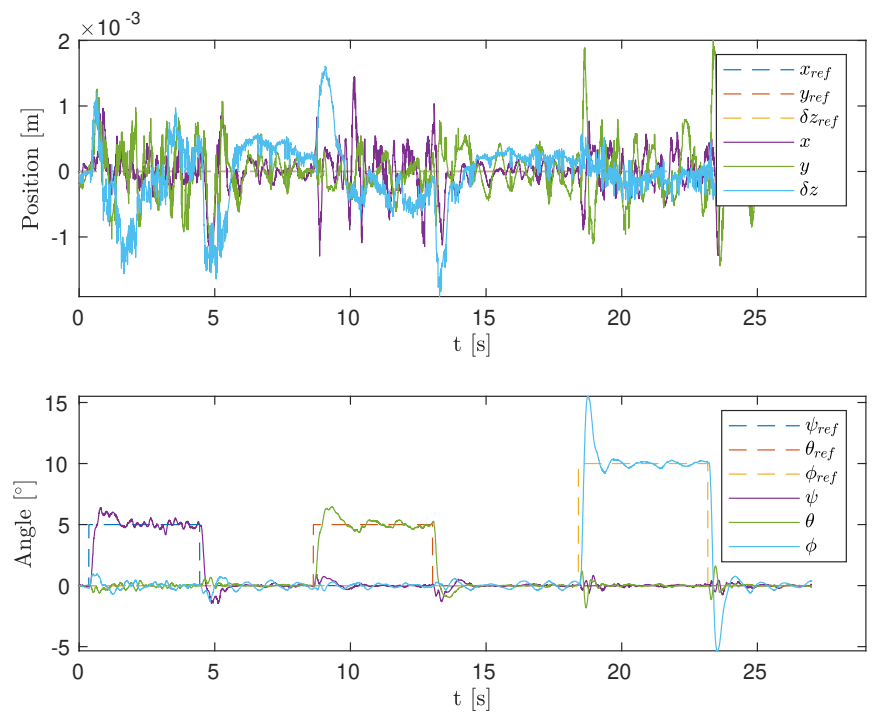

Fig. 7. Response to orientation steps.

obtained by the motion capture system is highly noisy and there is no available accelerometer on the robot, the acceleration is obtained using linear regression on a sliding window with 15 acquisitions of the AWG velocity. At the equilibrium position, the maximum acceleration is approximately $7 \mathrm{~m} \mathrm{~s}^{-2}$ in $x$ and $y$ directions and $6 \mathrm{~m} \mathrm{~s}^{-2}$ in $z$ direction.

The AWG can achieve higher accelerations at positions where the gravity is not fully compensated by the spring. At $60 \mathrm{~cm}$ above the equilibrium position, the maximum downward acceleration is $1.33 g$. Note that a standard suspended CDPR cannot achieve a downward acceleration higher than $1 g$, which occurs during free fall.

TABLE III

STEP RESPONSES

\begin{tabular}{lrrrrrr}
\hline & $x$ & $y$ & $z$ & $\psi$ & $\theta$ & $\phi$ \\
\hline Rise time [s] & 0.09 & 0.08 & 0.09 & 0.14 & 0.18 & 0.09 \\
Overshoot [\%] & 54.20 & 67.23 & 45.74 & 28.02 & 29.45 & 55.10
\end{tabular}

It is possible to reach an even higher acceleration by going beyond the static workspace of the robot and therefore minimizing the gravity compensation from the spring [22].

3) Disturbance rejection: Carrying a load, for example during a pick and place task, results in a vertical force step disturbance. The rejection of such a disturbance is assessed by hanging a $0.5 \mathrm{~kg}$ weight to the bottom of the AWG, where the gripper should be placed, and cutting it off. The response is shown on Fig. 8, the disturbance happens at $t=0$. The maximum displacement is less than $2 \mathrm{~cm}$, and the disturbance is rejected in approximately $700 \mathrm{~ms}$.

Note that the load could also be included in the dynamic model and not be considered as a disturbance.
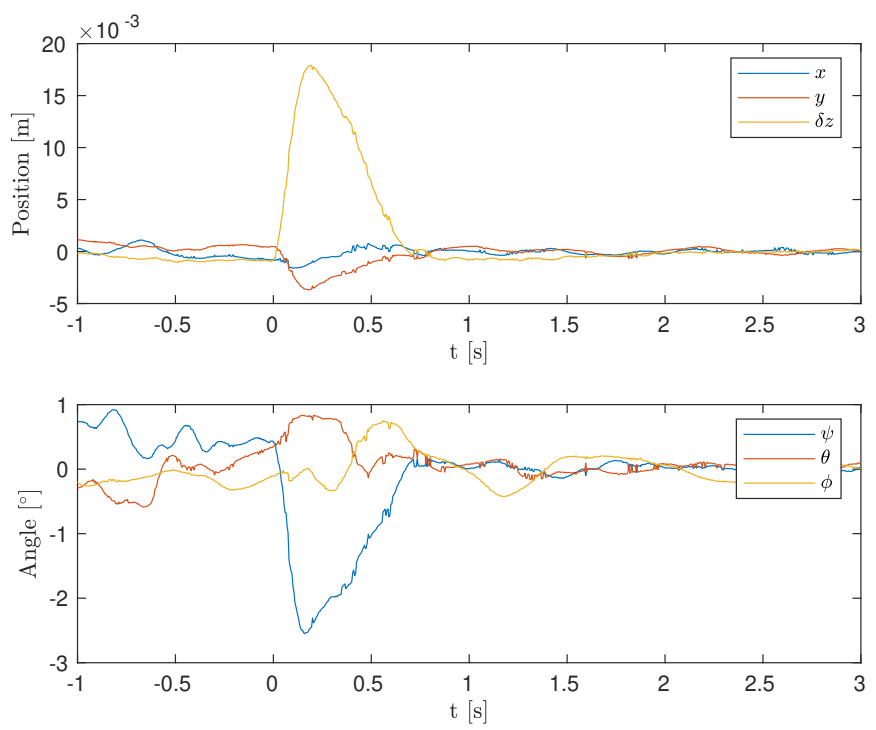

Fig. 8. Disturbance rejection.

\section{Energy performance}

The mechanical power $P_{m}$ developed by a propeller is the product of the rotational velocity $w$ by the drag torque $\tau_{d}=b w^{2}$, and so is proportional to the cubic rotational velocity: $P_{m}=b w^{3}$. The consumed electric power $P_{e}$ is the product of the battery voltage $U$ by the current $I$ delivered by the battery. The voltage $U$ depends on the charge of the battery and on its serial resistance, it is assumed constant. In steady state, that is when the rotational velocity $w$ is constant, the mechanical power and the electric power are equal up to a multiplicative constant corresponding to the efficiency. During a smooth trajectory, propeller velocities are considered quasi-static, so the power consumed for acceleration can be neglected. Therefore, the current $I$ is proportional to the cube of the rotational velocity $w$. Then, it is possible to simulate the energy consumption of the robot.

On a propulsion unit, since there is always one propeller rotating at the minimum speed, the total power consumption can be modeled efficiently as $P=c_{1} w^{3}+c_{2}$, with $c_{1}$ and $c_{2}$ constants to be identified. In Fig. 9 electric energy consumption measured on the dextAIR prototype and simulated consumption are compared for $c_{1}=2.05 e-8$ and $c_{2}=8.75$. The reference trajectory is the one used for the accuracy 
and repeatability tests with 10 cycles. Flat parts of the curve correspond to the equilibrium position, where the consumption is low.

For the same trajectory, the energy consumption of the same robot without the spring is $49 \mathrm{Wh}, 3.8$ times higher than dextAIR. This justifies the use of a spring to minimize energy consumption without increasing too much the mechanical complexity.

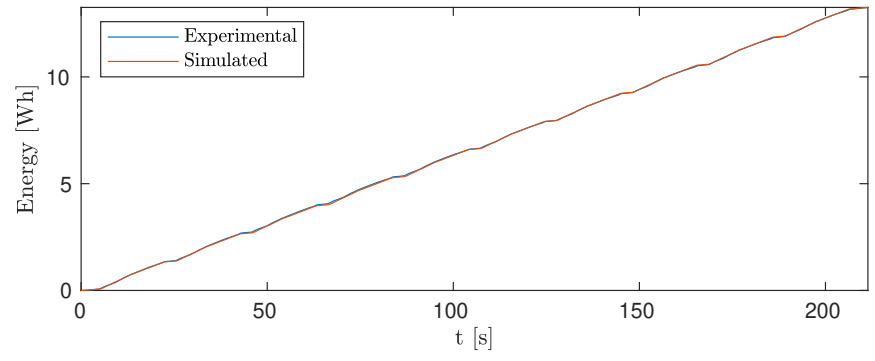

Fig. 9. Energy consumption of dextAIR robot.

\section{CONCLUSIONS AND FUTURE WORK}

In this paper, we present a novel Aerial Manipulator with Elastic Suspension. A computed torque controller is implemented and its stability is proven using singular perturbation theory. The performance characteristics are assessed experimentally.

We show that the AMES can have millimetric accuracy, fast dynamics and is more energy efficient than an untethered aerial manipulator.

The study of the static workspace suggests possible improvements of the mechanical design of the AMES to enhance significantly the workspace capabilities and thereby the energy efficiency. An optimization on propeller positioning can be considered to deal with the trade-off between dynamic performances and workspace.

\section{ACKNOWLEDGMENT}

We thank Luc Doppler and Quentin Boyer for their contribution to the development of the prototype.

\section{REFERENCES}

[1] F. Ruggiero, V. Lippiello, and A. Ollero, "Aerial Manipulation: A Literature Review," IEEE Robotics and Automation Letters, vol. 3, no. 3, pp. 1957-1964, 7 2018. [Online]. Available: https://ieeexplore.ieee.org/document/8299552/

[2] M. Tognon and A. Franchi, Theory and Applications for Control of Aerial Robots in Physical Interaction Through Tethers, ser. Springer Tracts in Advanced Robotics. Cham: Springer International Publishing. 2021, vol. 140. [Online]. Available: http://link.springer.com/10.1007/ 978-3-030-48659-4

[3] "Arrêté du 17 décembre 2015 relatif à la conception des aéronefs civils qui circulent sans personne à bord, aux conditions de leur emploi et aux capacités requises des personnes qui les utilisent." [Online]. Available: https://www.legifrance.gouv.fr/ affichTexte.do?cidTexte=JORFTEXT000031679906

[4] K. Zhang, P. Chermprayong, T. M. Alhinai, R. Siddall, and M. Kovac, "SpiderMAV: Perching and stabilizing micro aerial vehicles with bio-inspired tensile anchoring systems," in 2017 IEEE/RSJ International Conference on Intelligent Robots and Systems (IROS). IEEE, 9 2017, pp. 6849-6854. [Online]. Available: http: ///ieeexplore.ieee.org/document/8206606/
[5] Y. S. Sarkisov, M. J. Kim, D. Bicego, D. Tsetserukou, C. Ott, A. Franchi, and K. Kondak, "Development of SAM: cable-Suspended Aerial Manipulator *," in 2019 International Conference on Robotics and Automation (ICRA). IEEE, 5 2019, pp. 5323-5329. [Online]. Available: https://ieeexplore.ieee.org/document/8793592/

[6] G. El-Ghazaly, M. Gouttefarde, and V. Creuze, "Hybrid cable-thruster actuated underwater vehicle-manipulator systems: A study on force capabilities," in 2015 IEEE/RSJ International Conference on Intelligent Robots and Systems (IROS). IEEE, 9 2015, pp. 1672-1678. [Online]. Available: http://ieeexplore.ieee.org/document/7353592/

[7] H. Sellet, I. Khayour, L. Cuvillon, S. Durand, and J. Gangloff, "Active Damping of Parallel Robots Driven by Flexible Cables Using Cold-Gas Thrusters," in 2019 International Conference on Robotics and Automation (ICRA). IEEE, 5 2019, pp. 530-536. [Online]. Available: https://ieeexplore.ieee.org/document/8794061/

[8] I. Khayour, L. Cuvillon, C. Butin, A. Yiğit, S. Durand, and J. Gangloff, "Improving Disturbance Rejection and Dynamics of Cable Driven Parallel Robots with On-board Propellers," in 2020 IEEE/RSJ International Conference on Intelligent Robots and Systems (IROS). IEEE, 2020.

[9] A. Yiğit, G. Grappe, L. Cuvillon, S. Durand, and J. Gangloff, "Preliminary Study of an Aerial Manipulator with Elastic Suspension," in 2020 IEEE International Conference on Robotics and Automation (ICRA). IEEE, 5 2020, pp. 4287-4293. [Online]. Available: https://ieeexplore.ieee.org/document/9196942/

[10] M. Hamandi, F. Usai, Q. Sablé, N. Staub, M. Tognon, and A. Franchi, "Survey on Aerial Multirotor Design: a Taxonomy Based on Input Allocation," 2020. [Online]. Available: https: //hal.archives-ouvertes.fr/hal-02433405/document

[11] M. Tognon and A. Franchi, "Omnidirectional Aerial Vehicles With Unidirectional Thrusters: Theory, Optimal Design, and Control," IEEE Robotics and Automation Letters, vol. 3, no. 3, pp. 2277-2282, 72018. [Online]. Available: http://ieeexplore.ieee.org/document/8281444/

[12] W. Khan and M. Nahon, "Toward an Accurate Physics-Based UAV Thruster Model," IEEE/ASME Transactions on Mechatronics, vol. 18, no. 4, pp. 1269-1279, 8 2013. [Online]. Available: http://ieeexplore.iee.org/document/6523983/

[13] W. Chung, L.-C. Fu, and S.-H. Hsu, "Motion Control," in Springer Handbook of Robotics. Berlin, Heidelberg: Springer Berlin Heidelberg, 2008, pp. 133-159. [Online]. Available: http: //link.springer.com/10.1007/978-3-540-30301-5_7

[14] J. Begey, L. Cuvillon, M. Lesellier, M. Gouttefarde, and J. Gangloff, "Dynamic Control of Parallel Robots Driven by Flexible Cables and Actuated by Position-Controlled Winches," IEEE Transactions on Robotics, vol. 35, no. 1, pp. 286-293, 2 2019. [Online]. Available: https://ieeexplore.ieee.org/document/8521707/

[15] H. K. Khalil, Nonlinear Systems, 3rd ed. Upper Saddle River, New Jersey: Prentice Hall, 2002

[16] D. Brescianini and R. D'Andrea, “An omni-directional multirotor vehicle,” Mechatronics, vol. 55, pp. 76-93, 11 2018. [Online]. Available: https://linkinghub.elsevier.com/retrieve/pii/S0957415818301314

[17] S. Park, J. Lee, J. Ahn, M. Kim, J. Her, G.-H. Yang, and D. Lee, "ODAR: Aerial Manipulation Platform Enabling Omnidirectional Wrench Generation," IEEE/ASME Transactions on Mechatronics, vol. 23, no. 4, pp. 1907-1918, 8 2018. [Online]. Available: https://ieeexplore.ieee.org/document/8401328/

[18] A. Franchi and A. Mallet, "Adaptive closed-loop speed control of BLDC motors with applications to multi-rotor aerial vehicles," in 2017 IEEE International Conference on Robotics and Automation (ICRA). IEEE, 5 2017, pp. 5203-5208. [Online]. Available: http://ieeexplore.ieee.org/document/7989610/

[19] J. Gangloff, A. Yiğit, and M. Lesellier, "RPIt," 2020. [Online]. Available: https://github.com/jacqu/RPIt/

[20] Q. Jiang and C. M. Gosselin, "Maximal Singularity-Free Total Orientation Workspace of the Gough-Stewart Platform," Journal of Mechanisms and Robotics, vol. 1, no. 3, 8 2009. [Online]. Available: https://asmedigitalcollection.asme.org/mechanismsrobotics/article/doi/ 10.1115/1.3147200/454063/Maximal-SingularityFree-Total-Orientation

[21] "Manipulating industrial robots - Performance criteria and related test methods," NF ISO 9283, 2016

[22] S. Xiang, H. Gao, Z. Liu, and C. Gosselin, "Dynamic transition trajectory planning of three-DOF cable-suspended parallel robots via linear time-varying MPC," Mechanism and Machine Theory, vol. 146, p. 103715, 4 2020. [Online]. Available: https://linkinghub.elsevier.com/ retrieve/pii/S0094114X19323213 